\title{
Accuracy of Signs, Symptoms, and Hematologic Parameters for the Diagnosis of Infectious Mononucleosis: A Systematic Review and Meta-Analysis
}

\author{
Xinyan Cai, PhD, MSPH, Mark H. Ebell, MD, MS, and Lauren Haines
}

Background: The accuracy of individual symptoms, signs, and several easily obtainable hematologic parameters for diagnosing infectious mononucleosis (IM) still needs to be confirmed. Improving the diagnosis of IM based on the clinical findings could prompt physicians to identify better which patients need a diagnostic test for IM. This study performed a systematic review to determine the accuracy of symptoms, signs, and hematologic parameters in patients with suspected IM that used heterophile antibody test or viral capsid antigen tests as the reference standard.

Methods: The PubMed database was searched for all relevant articles. Two reviewers reviewed all studies in parallel and assessed the quality of the selected studies using the quality assessment of diagnostic accuracy studies 2 (QUADAS-2) criteria. The pooled measures of diagnostic performance were calculated by bivariate meta-analysis for each clinical finding, which included sensitivity, specificity, likelihood ratios, the diagnostic odds ratios, and the area under the receiver operating characteristic curve.

Results: Seventeen studies were included in our final analysis. The prevalence of IM ranged from $2.1 \%$ to $80 \%$ among prospective cohort studies. The presence of splenomegaly (positive likelihood ratio [LR+], 2.39; 95\% confidence interval $[\mathrm{CI}], 1.11-5.51)$, palatal petechiae $(\mathrm{LR}+, \mathbf{1 . 3 2 - 1 1 . 4 0})$, posterior cervical lymphadenopathy (LR+, 3.16; 95\% CI, 1.45-5.20), and axillary or inguinal cervical lymphadenopathy $(\mathrm{LR}+, 3.05$; 95 CI, 1.85-4.70) were moderately useful for ruling in IM. The most helpful hematologic parameters for ruling in IM include lymphocytes greater than $4 \times 10^{9} / \mathrm{L}$ and greater than $40 \%$ to $50 \%$, or atypical lymphocytes greater than $\mathbf{4 0 \%}$. A combination of lymphocytes greater than $\mathbf{5 0 \%}$ and atypical lymphocytes greater than $10 \%(\mathrm{LR}+, 50.40 ; 95 \% \mathrm{CI}, 8.43-162)$ was also found to be helpful to rule in disease. Most of the clinical findings have limited diagnostic value in ruling out the disease when absent.

Conclusions: Although most symptoms and signs were unhelpful, the likelihood of IM is appreciably increased by several examination findings. Hematologic parameters were more accurate than symptoms and signs. Since most clinical findings have limited diagnostic value in ruling out the disease, physicians should not rely on the absence of any individual symptom or clinical sign for ruling out IM. ( $J$ Am Board Fam Med 2021;34:1141-1156.)

Keywords: Evidence-Based Medicine, Family Practice, Infectious Mononucleosis, Meta-Analysis

\section{Background}

Infectious mononucleosis (IM) is a disease caused by the Epstein-Barr virus (EBV). IM is most

This article was externally peer reviewed.

Submitted 23 May 2021; revised 5 August 2021; accepted 9 August 2021.

From the Department of Epidemiology and Biostatistics, College of Public Health, University of Georgia, Athens, GA, USA (XC, MHE, LH).

Funding: None.

Conflict of interest: The authors have no relevant financial information or potential conflicts to disclose.

Corresponding author: Xinyan Cai PhD, MSPH, 108 Miller Hall, University of Georgia Health Sciences Campus, Athens, GA 30602, Phone: 803-261-1074 (E-mail: Xinyan. Cai@uga.edu). common among young adults, especially those aged 15 to 24 years, with the incidence rate ranging from 6 to 8 cases per 1000 person-years. ${ }^{1}$ The most common symptoms and signs among IM patients include sore throat, lymph node enlargement, fever, tonsillar enlargement, pharyngeal inflammation, fatigue, and rashes. ${ }^{2-4}$ However, the diagnostic accuracy of the symptoms and signs of IM is inconsistent among studies. ${ }^{5,6}$

A recent systematic review ${ }^{2}$ of the diagnostic accuracy for individual symptoms and signs found that the absence of sore throat (sensitivity, 0.81 ; negative likelihood ratio [LR-], 0.51-0.62), headache (sensitivity, 0.66; LR-, 0.63-0.73), or any 
lymphadenopathy (sensitivity, 0.91; LR-, 0.23-0.44) reduces the likelihood of IM, and the presence of posterior cervical adenopathy (specificity, 0.87; positive likelihood ratio [LR+], 1.6-5.9) or splenomegaly (specificity, 0.71-0.99; LR+, 1.9-6.6) increases the likelihood of IM. From this review, only 2 studies $^{5,6}$ reported on both the sensitivity and specificity of the symptoms and clinical signs and other studies used patients with known IM, which can only determine the sensitivity. Therefore, the accuracy of the clinical findings still needs to be confirmed.

Previous studies ${ }^{4,6,7}$ also found that several easily obtainable hematologic parameters, including complete blood counts (CBC) and leukocyte differential counts, are helpful in diagnosing IM among clinically suspected patients. The presence of atypical lymphocytosis has been shown to significantly increase the likelihood of IM, and a high percentage of lymphocytes also helps to increase the likelihood of IM when combined with atypical lymphocytosis. ${ }^{6,8}$ Patients presenting with monocytosis were also found to have a higher likelihood of IM. $^{8,9}$ Thus far, there is only 1 systematic review ${ }^{2}$ focused on the accuracy of clinical presentations or hematologic parameters for the diagnosis of IM. Most of the included studies from the previous review were old, and some prospective cohort studies or case series were not included in that analysis.

In the present analysis, we performed an updated meta-analysis of the accuracy of the symptoms, clinical signs, and hematologic parameters for the diagnosis of IM. We also did a subgroup analysis of the commonly reported risk factors according to reference standard tests and various patient settings to assess potential sources of heterogeneity.

\section{Method}

This study was registered with the PROSPERO database (\#CRD 42021246280), and our study followed the preferred reporting items for systematic reviews and meta-analyses (PRISMA) protocol for guidance. ${ }^{10}$

\section{Search Strategy and Selection Criteria}

The PubMed database was searched for articles published through 30 September 2020, with no restrictions on language. The following search concepts were used involving all possible elements: "signs, symptoms, and diagnostic tests"; "infectious mononucleosis or Epstein-Barr virus"; and "accuracy of diagnosis" linked by the Boolean operator "AND." The filters "has abstract" and "human" were applied to the search. The complete search strategy for the PubMed database is shown in Appendix S1. We also manually searched for relevant articles from the reference lists of the included studies. This meta-analysis included only published and peer-reviewed studies.

All titles and abstracts were screened in parallel by 2 independent reviewers $(\mathrm{XC}, \mathrm{LH})$. If there was any uncertainty regarding eligibility, a third reviewer (ME) was consulted. The full-text article for each abstract was then independently reviewed by 2 reviewers $(\mathrm{XC}, \mathrm{LH})$, and the third reviewer (ME) was responsible for resolving discrepancies among the reviewers through a consensus discussion at this stage.

\section{Inclusion Criteria}

This systematic review and meta-analysis included studies that performed original data collection and provided sufficient information to construct a $2 \times 2$ table for the diagnostic accuracy of at least 1 symptom, clinical sign, or hematologic parameter (eg, WBC, lymphocytes, atypical lymphocytes, monocytes) for IM against a reference standard test. In this meta-analysis, we limited the reference standard tests to commercial heterophile antibody tests (eg, Monospot test), viral capsid antigen (VCA)-specific tests (eg, the indirect immunofluorescence test [IFA], or an enzyme-linked immunosorbent assay [EIA]) for the detection of EBV infection.

Eligible studies included cohort studies with patients suspected to have IM or EBV infection and had sufficient information to calculate both the sensitivity and specificity of the test or case series that had sufficient data to calculate the sensitivity. Only prospective cohort studies or case series (to calculate sensitivity) were included for the study of symptoms and signs. At the same time, retrospective cohort studies were also included for the study of hematologic parameters. Data were collected from patients in any age group. The same reference standard test had to be performed for all patients. We included studies with both inpatient and outpatient settings with no restrictions on languages, country, or year of publication.

We excluded review articles, individual case reports, commentaries, editorials, case-control studies, retrospective cohort studies for the study of 
symptoms or signs, modeling studies, and studies with sample sizes less than 5. A study was also excluded if it focused on immunodeficient subjects or subjects with a special condition (ie, another acute illness consistent with EBV-related IM, transplant recipients, or HIVpositive subjects); studies of IM primarily caused by toxoplasmosis or cytomegalovirus infection were eliminated from consideration as well. A list of excluded studies and a $\log$ of the respective reasons can be provided by the authors on request.

\section{Data Abstraction}

Two investigators (XC, LH) were responsible for extracting the aggregate study data. The following data were extracted from studies: study characteristics (country, year of recruitment, setting), study populations (mean or median age, gender, sample size, inclusion and exclusion criteria), the index tests being studied, the reference standard test being used, and the results from each study to construct $2 \times 2$ tables. If the absolute numbers were not reported, they were estimated based on the total number of patients, the sensitivity, and the specificity.

\section{Quality Assessment}

Two reviewers (XC, LH) assessed the quality of the selected studies using the quality assessment of diagnostic accuracy studies 2 (QUADAS-2) tool. The 4 key domains of the QUADAS-2 tool include patient selection, evaluation of the index test performance, evaluation of the reference test performance, and flow of patients through the study with corresponding timing. ${ }^{11} \mathrm{We}$ defined low, unclear, and high risk of bias for each domain according to the study quality. A third reviewer (ME) was responsible for resolving any disagreements during the quality assessment. We then displayed the proportion of studies by differing degrees of risk of bias. ${ }^{11}$ The full QUADAS-2 instruments adapted for our study and their corresponding definition questions are shown in Appendix S2.

\section{Analytic Strategy}

In our primary analysis, we calculated summary estimates of the diagnostic accuracy of the symptoms, clinical signs, and hematologic parameters of IM. We first extracted the data needed for the construction of $2 \times 2$ contingency tables that compared the diagnostic performance of each clinical findings with the reference standard test. Then, we calculated the pooled measures of diagnostic performance for each element, such as sensitivity, specificity, positive $(\mathrm{LR}+)$ and negative likelihood ratios (LR-), and diagnostic odds ratios (DORs), with their corresponding 95\% confidence intervals. The DOR is calculated by LR+ divided by LR-, where a higher DOR indicates a better diagnostic accuracy for the underlying test to detect IM in our study. ${ }^{12}$ If there were only data from a single study, the point estimate and 95\% confidence intervals [CIs] are presented; if there were data from 2 studies, ranges were presented; if there were data from 3 or more studies, the summary estimates were calculated based on a bivariate meta-analysis, and the area under the receiver operating characteristics curve (AUC) was determined. Similar cutoffs for the same index test were combined if clinically reasonable; for example, white blood cell counts greater than $9.5 \times 10^{9} / \mathrm{L}, 10 \times 10^{9} / \mathrm{L}$, and $10.5 \times 10^{9} / \mathrm{L}$ were combined into a single cutoff of "greater than 9.5 to $10.5 \times 10^{9} / \mathrm{L}$."

We imported the data into $\mathrm{R}$ and performed a bivariate analysis on 3 or more studies for the same index test with same or similar cutoffs using the mada package. ${ }^{13,14}$ The AUCs and DORs with 95\% confidence intervals (CIs) were also calculated using this package. If only 1 study described the accuracy of a test, we used the diagti procedure in Stata 15.1 (Stat-Corp) to estimate the overall accuracy with $95 \%$ CIs.

We also looked for the presence of threshold effects during the analysis to check whether the sensitivity decreased and the specificity increased with increasing diagnostic cutoffs or thresholds. ${ }^{15}$ The threshold effect was determined by visually inspecting the receiver operating characteristics curves (ROCs) stratified by the cutoffs of the index test. If a significant threshold effect existed, it was generally not feasible to calculate or report 1 summary statistic for the diagnostic performance of each test, and we instead presented the summary estimate of diagnostic accuracy of the test stratified by the cutoff value.

Finally, subgroup analyses were performed on selected symptoms, signs, and hematologic parameters by the reference standard test chosen (heterophile antibody test vs VCA-specific test) and the study settings (inpatient vs outpatient). 


\section{Patient and Public Involvement}

Patients did not develop the research, measure the research outcomes, conduct the research, or prepare the manuscript.

\section{Results}

\section{Study Characteristics}

We identified 1173 abstracts in our initial search and 14 from the review of the reference lists. Thus, the authors reviewed a total of 1187 abstracts, of which 191 were reviewed in full, and 17 studies met our inclusion and exclusion criteria and were included in our quantitative analysis. The search process is summarized in Figure 1.

The characteristics of each included study are summarized in Table 1. The number of patients studied ranged from 25 to 1000 . The average age of the participants was between 4 and 32 years, with $37 \%$ to $72 \%$ being female. There were 8 studies set in the United States, ${ }^{3,5,16-21} 1$ each in Canada, ${ }^{8}$ Israel, ${ }^{6}$ and Saudi Arabia, ${ }^{22}$ and the remainder were from Europe. We identified 12 studies with information on the accuracy of clinical symptoms and signs, of which 8 were prospective cohort studies, 5,6,16,19,22-25 and 4 were case series; $3,17,21,26$ only the sensitivity of the symptoms and signs could be calculated from these case series. We also identified 10 studies for the study of hematologic parameters, ${ }^{5,6,8,9,18-20,22,24,27}$ of which 3 were retrospective studies. ${ }^{9,18,27}$ Seven studies used the heterophile antibody test as the reference standard test for IM, ${ }^{5,8,9,18,21,25,27}$ and 4 studies used the VCA-specific test as the reference standard; ${ }^{16,19,20,24}$ 6 studies used both the heterophile antibody test and VCA-specific test as the confirmatory test for IM. ${ }^{3,6,17,22-23,26}$ The prevalence of IM ranged from $2.1 \%^{5}$ to $80 \%{ }^{16}$ in the prospective cohort studies. Six prospective cohort studies ${ }^{5,9,18,20,24,25}$ had a prevalence of IM below $50 \%$ and $2^{5,9}$ were below $10 \%$.

\section{Quality Assessment}

The study quality was assessed using the QUADAS-2 framework. Of the 17 studies, 3 were judged to have a high risk of bias, ${ }^{16,21,25} 5$ a moderate risk of bias, ${ }^{17-20,22}$ and the remainders were judged to have a low risk of bias (Table 2). A detailed description of the quality assessment using the QUADAS tool is described in Appendix S2.

\section{Accuracy of the Clinical Findings}

The accuracy of the clinical symptoms, clinical signs, and hematologic parameters is summarized in Table 3; the full dataset with individual study level data for each symptom or sign is provided in Appendix S6, and the individual level data for each hematologic parameter is provided in Appendix S7. The clinical symptoms significantly associated with IM based on likelihood ratios were headache (LR+, 1.19; 95\% CI, 1.01-1.45; LR-, 0.72; 95\% CI: $0.50-0.98)$ and sore throat $(\mathrm{LR}+, 1.12 ; 95 \%$ CI, 1.01-1.25; LR-, 0.67; 95\% CI, 0.41-0.99). No other symptoms had likelihood ratios significantly associated with the diagnosis of IM.

Regarding signs, presence of splenomegaly (LR+, 2.39; 95\% CI, 1.11-5.51; LR-, 0.66; 95\% CI, 0.50-0.84), palatal petechiae (LR+, 1.32-11.4; LR-, 0.57-0.94), and any lymphadenopathy (LR+, 1.26; 95\% CI, 1.05-1.65; LR-, 0.37; 95\% CI, 0.20 $0.67)$ significantly increased the likelihood of IM when present and reduced the likelihood of IM when absent. The absence of lymphadenopathy was the most accurate to rule out IM, and it had the highest AUC at 0.81 . The presence of any lymphadenopathy (LR+, 1.26; 95\% CI, 1.05-1.65; LR-, 0.37 ; 95\% CI, 0.20-0.67), posterior cervical lymphadenopathy and axillary (LR+, 3.16; 95\% CI, 1.455.20; LR-, 0.68; 95\% CI, 0.41-0.93) or inguinal lymphadenopathy $(\mathrm{LR}+, 3.05$; 95\% CI, 1.85-4.70; LR-, 0.67; 95\% CI, 0.3-0.91) were more helpful for ruling in than for ruling out IM. However, the likelihood ratios for posterior cervical lymphadenopathy and axillary or inguinal lymphadenopathy were calculated solely based on a single study with 709 IM susceptible patients. Pharyngitis had the highest sensitivity and was reported in 2 case series (sensitivity, 0.94; 95\% CI, 0.68-0.99). No data were available on the specificity of pharyngitis as it was usually a criterion for study entry; therefore, the likelihood ratios for pharyngitis could not be determined. Fever (measured temperature $\geq 37$ to $38.0^{\circ} \mathrm{C}$ ) was commonly reported but had little discriminatory value; this might be because fever was usually required as an entrance criterion for the studies.

Hematologic parameters were more accurate in diagnosing IM than symptoms and signs (Table 3). Based on the analysis, absolute lymphocyte counts greater than $4 \times 10^{9} / \mathrm{L}$ increased the likelihood of IM (LR+, 10.20; 95\% CI, 4.79-16.0). The likelihood of IM was also increased by an increasing percentage of atypical lymphocytes, from atypical lymphocytes greater than 10\% (LR+, 8.97; 95\% CI, 3.39-19.5) to atypical lymphocytes greater than 
Figure 1. PRISMA flow diagram describing the search process.

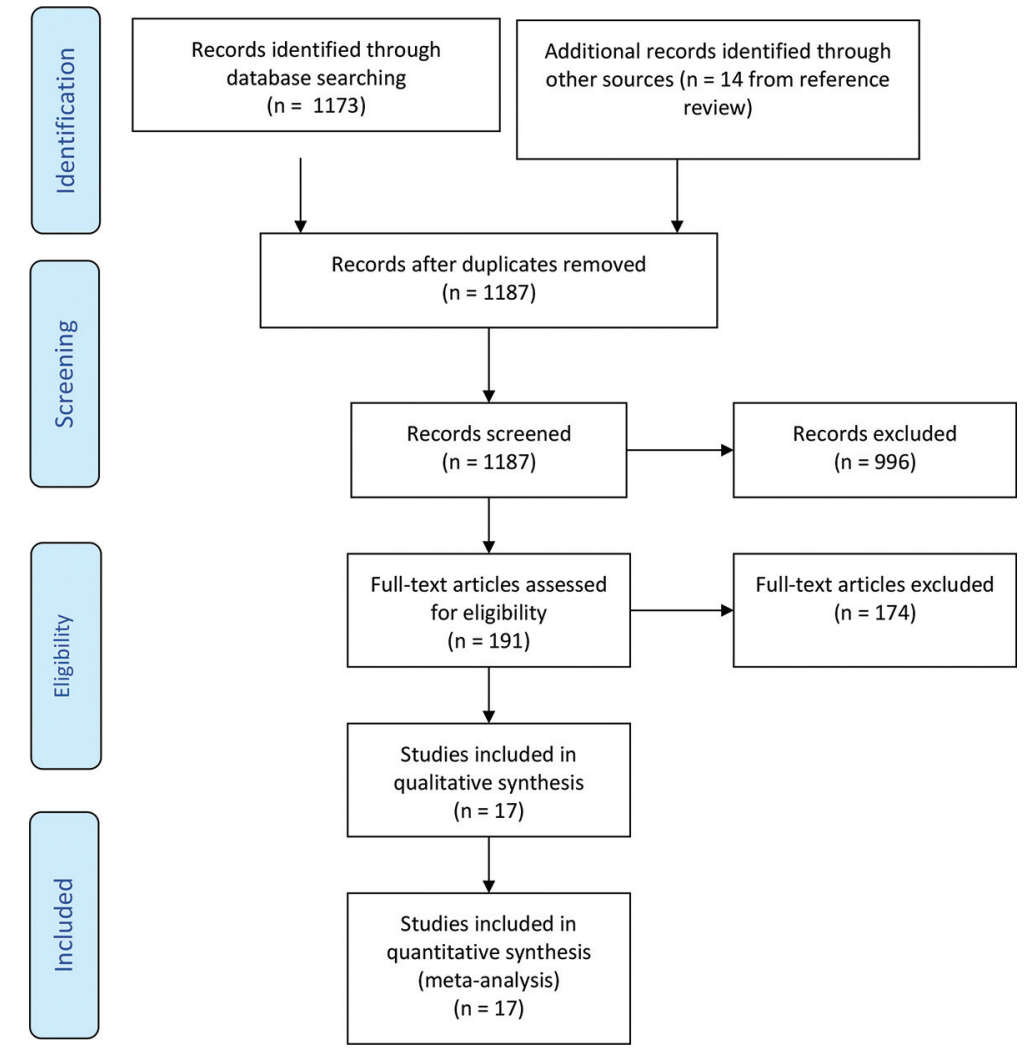

40\% (LR+, 50.3; 95\% CI, 38.6-64.1). The ability to rule out IM with different percentages of atypical lymphocytes was similar: the negative likelihood ratio ranged from 0.45 (95\% CI, 0.38-0.52) to 0.75 (95\% CI, 0.68-0.82). Our analysis also found that higher cutoff values for the percentage of lymphocytes had a stronger ability to rule in IM and a weaker ability to rule out disease. Our analysis also found that patients with a combination of lymphocytes greater than $50 \%$ and atypical lymphocytes greater than $10 \%$ would be more accurately ruling in IM (LR+, 50.4; 95\% CI, 8.43-162) compared with their individual effect. Other hematologic parameters associated with IM included monocytes greater than $1 \times 10^{9} / \mathrm{L}(\mathrm{LR}+, 1.46-7.89$, LR-, 0.11$0.98)$, leukocytes greater than $5 \times 10^{9} / \mathrm{L}(\mathrm{LR}+$, $1.05-1.36$, LR-, $0.15-0.70)$ or $10 \times 10^{9} / \mathrm{L}(\mathrm{LR}+$, 2.55; 95\% CI, 1.58-3.99; LR-, 0.68; 95\% CI, 0.56$0.82)$.

The diagnostic odds ratio (DOR) estimates the overall diagnostic accuracy of each index test. Based on the results, the highest DORs for the diagnosis of IM were atypical lymphocytes greater than $40 \%$ (DOR, 355; 95\% CI, 7.43-622), followed by a combination of atypical lymphocytes greater than $10 \%$ and lymphocytes greater than 50\% (DOR, 81.2; 95\% CI, 19.1-216), and then absolute lymphocyte counts greater than $4 \times 10^{9} / \mathrm{L}$ (DOR, 30.6; 95\% CI, 6.53-99.4). Posterior cervical lymphadenopathy (DOR, 5.18; 95\% CI, 1.55-12.6) had the best DOR compared with other individual symptoms and signs.

Summary ROC curves for the percentage of lymphocytes and atypical lymphocytes by cutoff values are shown in Figure 2. The differences in accuracy are related to the differences in the cutoffs, which indicate the threshold effects. Therefore, we reported an estimate of these hematologic parameters separately by cutoff values. The summary ROC curves using different reference standard tests for the lymphocytes greater than $50 \%$ and the atypical lymphocytes greater than $10 \%$ are shown in Figure 3. For the subgroup analysis, we found that the symptom of sore throat was relatively more specific in the outpatient setting than hospitalized patients, and the sensitivity of lymphocytes greater than $50 \%$ was higher in the outpatient setting than in the inpatient setting. However, these findings were 


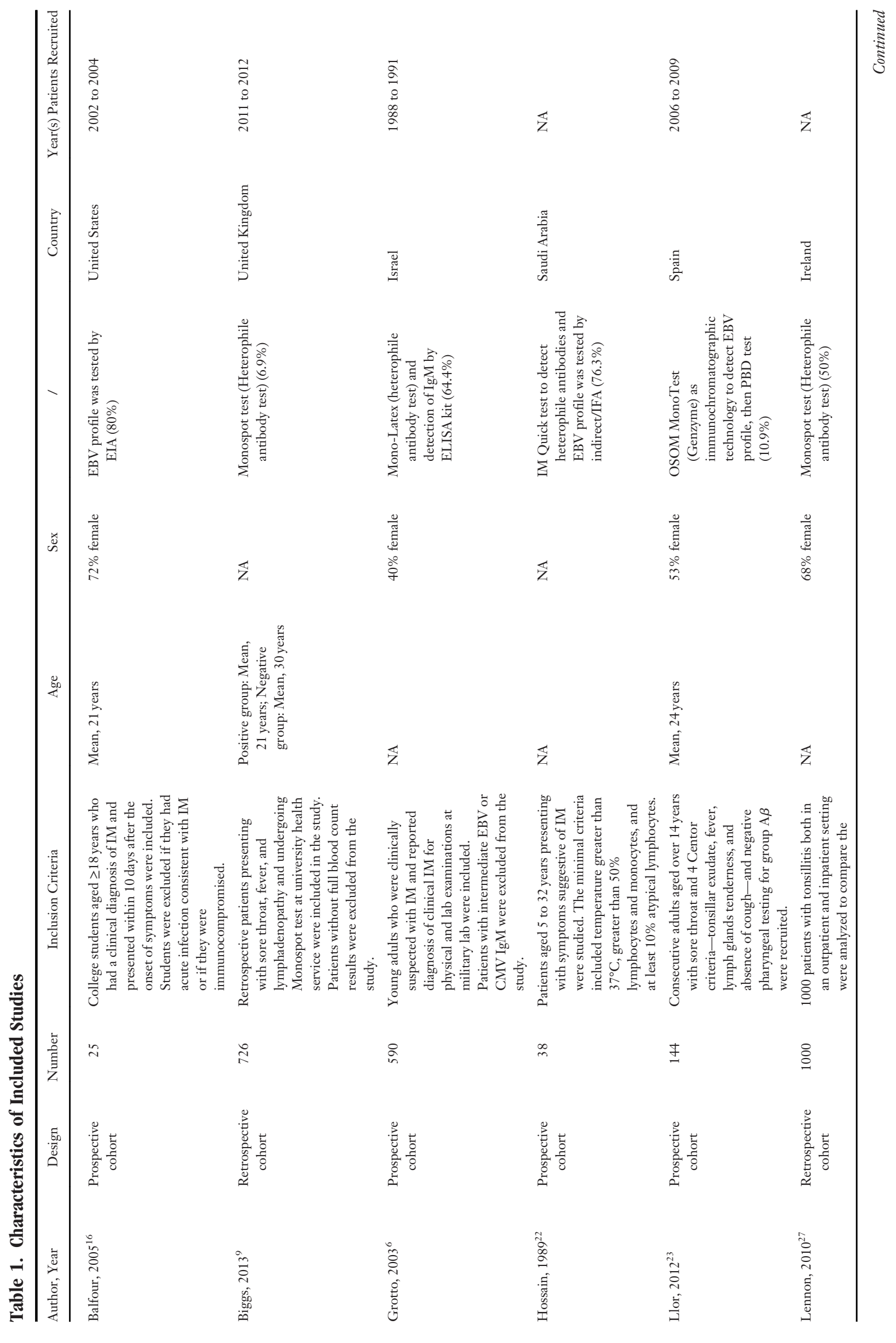

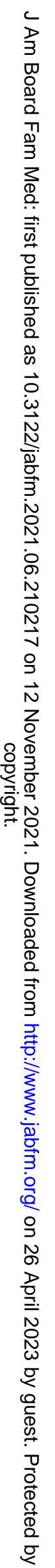




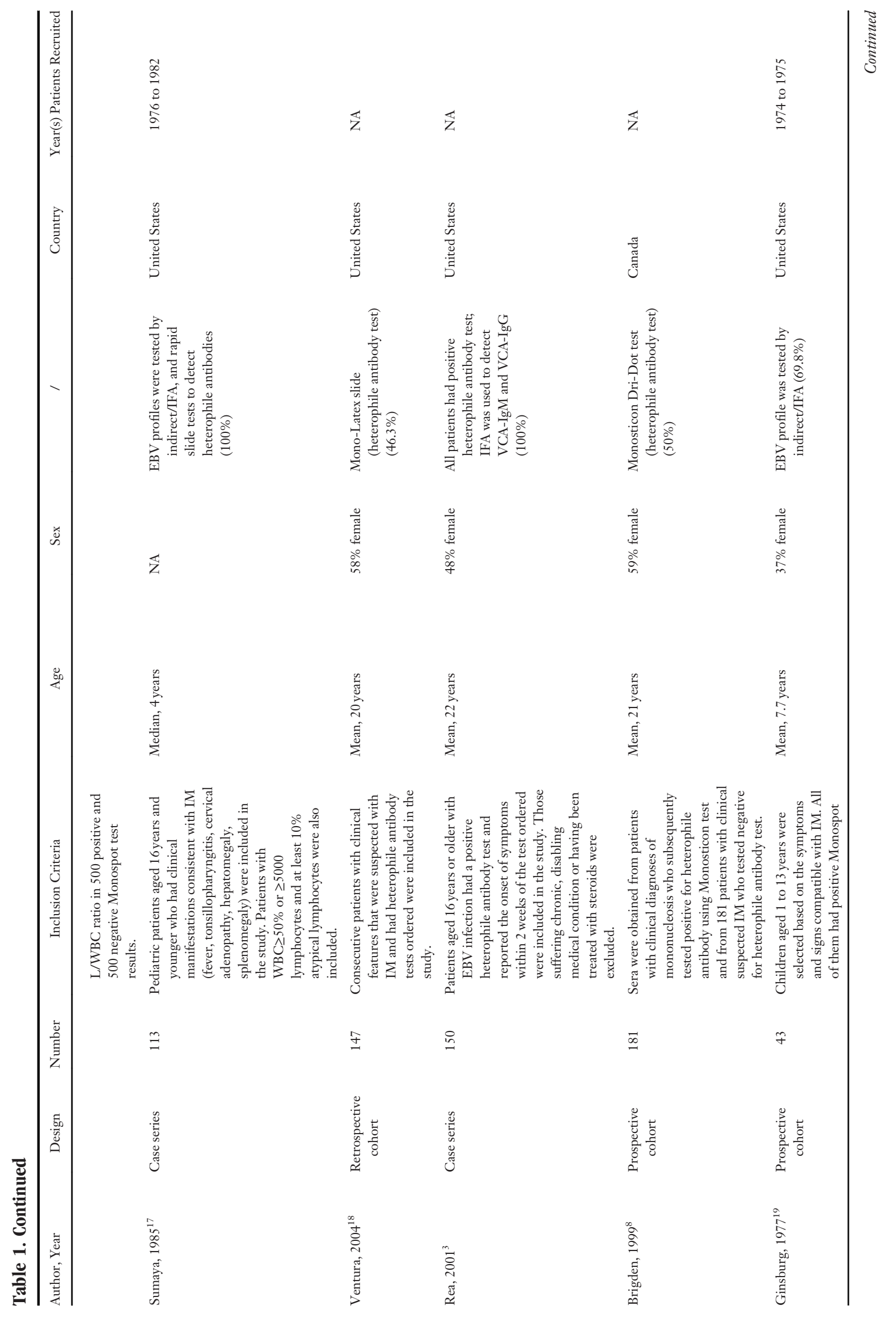




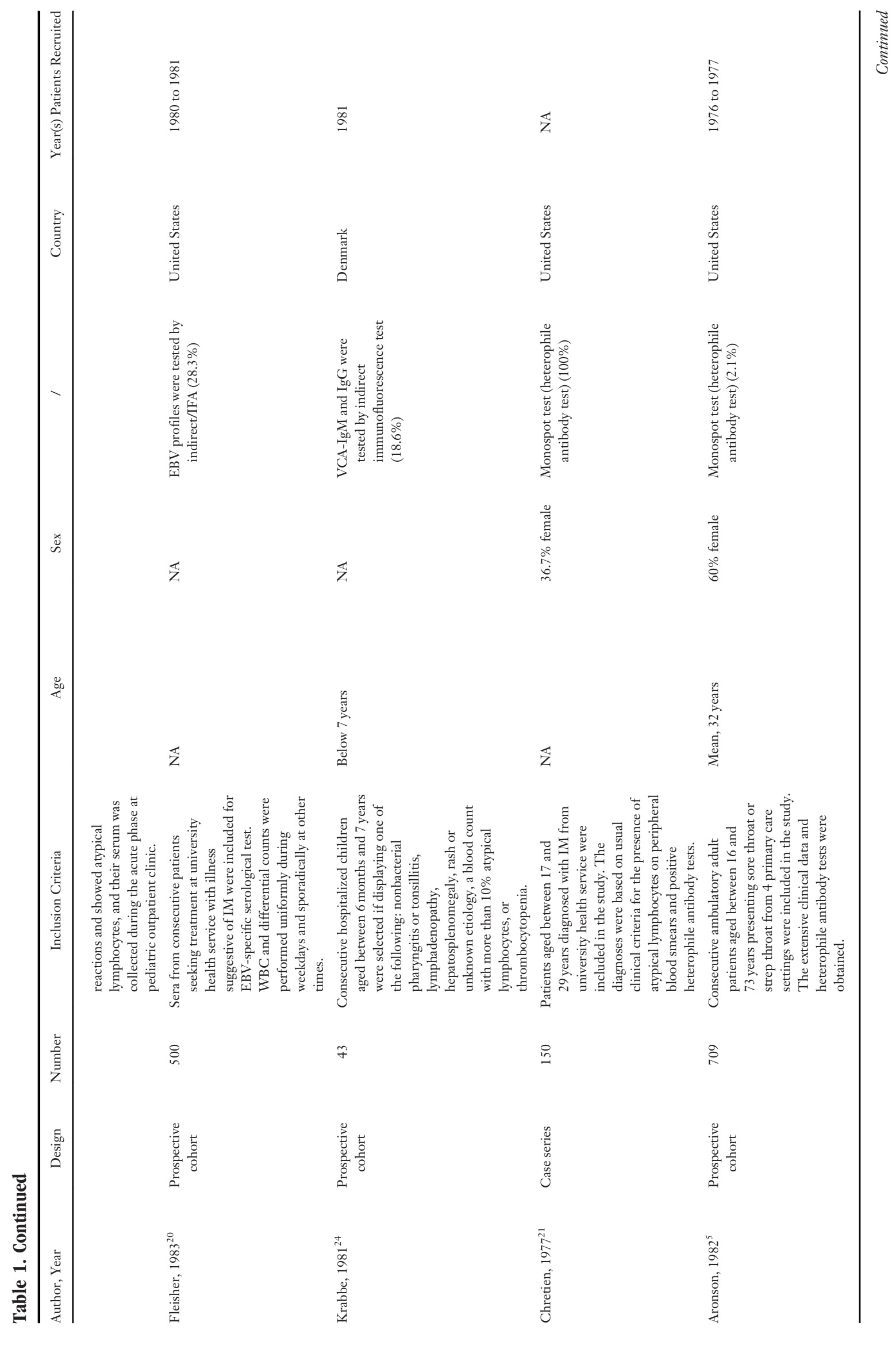




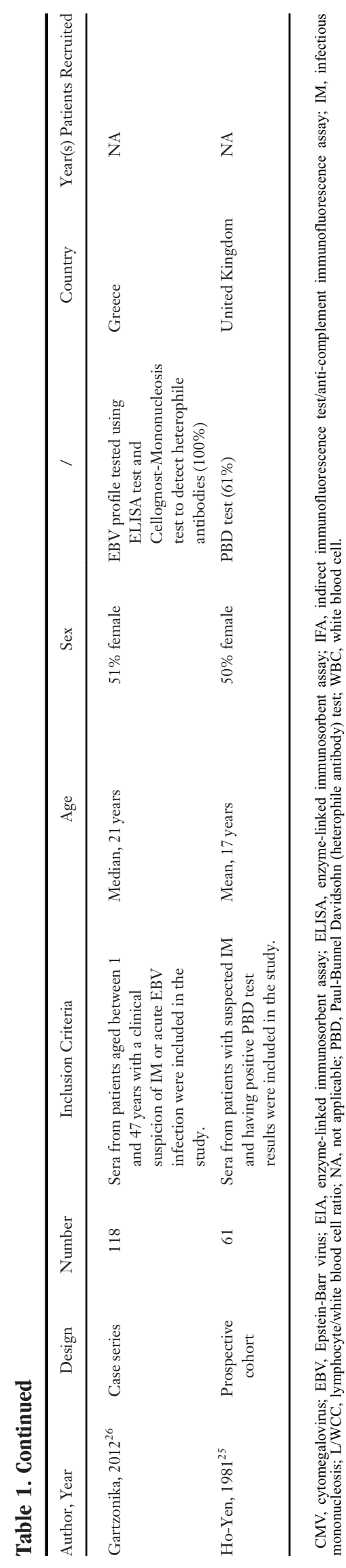

limited by the number of studies. No clear pattern for other clinical presentations or hematologic parameters was found regarding the standard reference test chosen (Appendix S3) or study settings (Appendix S4) based on visualizing the ROC curves. There were also no significant patterns for the hematologic parameters regarding different study designs based on the ROC curves (Appendix S5).

\section{Discussion}

This study is an updated meta-analysis on the individual symptoms, clinical signs, or hematologic parameters for predicting IM. Eight new studies with data for several symptoms and signs and 3 new articles about hematologic parameters that were not included in previous review ${ }^{2}$ were included in our analysis, supporting the need for an updated review.

There is a wide variation in the prevalence of IM among our included studies, with the range between $2.1 \%$ and $80 \%$ for patients with IM. The varying prevalence of IM is likely due to the different study settings and inclusion criteria. The study ${ }^{5}$ with a prevalence of $2.1 \%$, had very broad inclusion criteria, which comprised all patients aged between 16 to 73 years with sore throat or strep throat. The study $^{22}$ having the highest prevalence $(80 \%)$ had more restrictive inclusion criteria: clinical symptoms suggestive of IM, lymphocytes greater than $50 \%$, and atypical lymphocytes greater than $10 \%$.

The clinical history and physical examination are critical components of the evaluation of patients with suspected IM, and knowledge of the clinical signs and symptoms associated with IM would help physicians to decide whether diagnostic testing for IM is necessary. Based on the DORs for overall discrimination, the presence of splenomegaly $(\mathrm{LR}+$, 2.39; 95\% CI, 1.11-5.51), palatal petechiae (LR+, 1.32-11.4), posterior cervical lymphadenopathy $(\mathrm{LR}+, 3.16$; 95\% CI, 1.45-5.20), and axillary or inguinal lymphadenopathy (LR+, 3.05, 95 CI: 1.854.70) were moderately useful for ruling in IM. Therefore, patients presenting with 1 of these clinical signs could prompt physicians to order a diagnostic test for IM. Although the presence of posterior cervical lymphadenopathy and axillary or inguinal lymphadenopathy have shown to have moderate diagnostic values, they were based on a single study with 709 suspected patients. Thus, the 
Table 2. Overview of Study Quality*

\begin{tabular}{lccccc}
\hline Study, Year & Patient Selection & Index Test & Reference Std & Flow \& Timing & Overall \\
\hline Biggs, 2013 & $\mathrm{L}$ & $\mathrm{L}$ & $\mathrm{L}$ & $\mathrm{L}$ & $\mathrm{L}$ \\
Grotto, 2003 & $\mathrm{L}$ & $\mathrm{L}$ & $\mathrm{L}$ & $\mathrm{L}$ & $\mathrm{L}$ \\
Llor, 2012 & $\mathrm{L}$ & $\mathrm{L}$ & $\mathrm{L}$ & $\mathrm{L}$ & $\mathrm{L}$ \\
Lennon, 2010 & $\mathrm{L}$ & $\mathrm{L}$ & $\mathrm{L}$ & $\mathrm{L}$ & $\mathrm{L}$ \\
Rea, 2001 & $\mathrm{L}$ & $\mathrm{L}$ & $\mathrm{L}$ & $\mathrm{L}$ & $\mathrm{L}$ \\
Brigden, 1999 & $\mathrm{L}$ & $\mathrm{L}$ & $\mathrm{L}$ & $\mathrm{L}$ & $\mathrm{L}$ \\
Krabbe, 1981 & $\mathrm{L}$ & $\mathrm{L}$ & $\mathrm{L}$ & $\mathrm{L}$ & $\mathrm{L}$ \\
Aronson, 1982 & $\mathrm{L}$ & $\mathrm{L}$ & $\mathrm{L}$ & $\mathrm{L}$ & $\mathrm{L}$ \\
Gartzonika, 2012 & $\mathrm{L}$ & $\mathrm{L}$ & $\mathrm{L}$ & $\mathrm{L}$ & $\mathrm{L}$ \\
Hossain, 1989 & $\mathrm{L}$ & $\mathrm{H}$ & $\mathrm{L}$ & $\mathrm{L}$ & $\mathrm{M}$ \\
Sumaya, 1985 & $\mathrm{H}$ & $\mathrm{L}$ & $\mathrm{L}$ & $\mathrm{L}$ & $\mathrm{M}$ \\
Ventura, 2004 & $\mathrm{L}$ & $\mathrm{H}$ & $\mathrm{L}$ & $\mathrm{L}$ & $\mathrm{M}$ \\
Ginsburg, 1977 & $\mathrm{U}$ & $\mathrm{H}$ & $\mathrm{L}$ & $\mathrm{H}$ & $\mathrm{M}$ \\
Fleisher, 1983 & $\mathrm{L}$ & $\mathrm{L}$ & $\mathrm{H}$ & $\mathrm{L}$ & $\mathrm{M}$ \\
Chretien, 1977 & $\mathrm{L}$ & $\mathrm{H}$ & $\mathrm{H}$ & $\mathrm{H}$ & $\mathrm{H}$ \\
Ho-Yen, 1981 & $\mathrm{L}$ & $\mathrm{H}$ & $\mathrm{H}$ & $\mathrm{L}$ & $\mathrm{H}$ \\
Balfour, 2005 & $\mathrm{L}$ & & & & \\
\hline
\end{tabular}

${ }^{*} \mathrm{~L}=0, \mathrm{M}=1$, and $\mathrm{H}=2+$ with high likelihood of bias.

diagnostic accuracy of these clinical signs should be further evaluated in the future to get more precise estimates. Most of the individual clinical findings have limited diagnostic value in ruling out the disease; only the absence of any lymphadenopathy (LR-, 0.37; 95\% CI, 0.20-0.67) was moderately helpful to rule out IM. Therefore, physicians should not rely on the absence of any individual symptom or clinical sign for ruling out IM.

The hematologic parameters were more accurate for diagnosing IM compared with the patients' symptoms and signs. Lymphocytes greater than $4 \times 10^{9} / \mathrm{L}$, monocytes greater than $1 \times 10^{9} / \mathrm{L}$, leukocytes greater than $5 \times 10^{9} / \mathrm{L}$ or $10 \times 10^{9} / \mathrm{L}$, and higher percentages of lymphocytes and atypical lymphocytes all significantly increase the likelihood of IM. We also found that the combination of lymphocytes greater than $50 \%$ and atypical lymphocytes greater than $10 \%(\mathrm{LR}+, 50.40 ; 95 \% \mathrm{CI}$, $8.43-162.00)$ are very useful to rule in disease.

\section{Clinical Implications}

Compared with the previous review, ${ }^{2}$ we found additional studies that addressed lymphadenopathy, sore throat, headache, and splenomegaly, allowing us to make a more precise summary estimate of the accuracy for these symptoms and signs.
A previous study ${ }^{17}$ found that children less than 4 years with IM are more likely to have rashes, abdominal pain, and neurologic problems than adolescents and older adults. For adolescents, the most common symptoms of IM include headache, sore throat, and fatigue, and they were less likely to have diarrhea, rashes, and jaundice than other age groups. ${ }^{6}$ The adults with IM were more likely to have fatigue and sore throat and less likely to have myalgia or arthralgia. ${ }^{2}$ Older people are more likely to have fatigue and body pain and rarely have a sore throat. ${ }^{28}$ Acute infection of EBV for young children is usually either asymptomatic or expressed by mild upper respiratory tract symptoms, while for older patients (aged greater than 40 years), pharyngitis and cervical lymphadenopathy are less frequent myalgia is prominent. ${ }^{29,30}$ However, there were not enough prospective cohort studies for IM diagnosis in older adults; therefore, we did not conduct a stratified meta-analysis on the symptoms and signs based on the different age groups.

According to an Australian study, the pretest probability of IM is approximately $8 \%$ for adolescents aged 16 to 20 years with a sore throat. ${ }^{31}$ Based on our findings, the presence of splenomegaly $(\mathrm{LR}+, 2.39)$ would increase the probability of IM from $8 \%$ to $17 \%$, and posterior cervical lymphadenopathy $(\mathrm{LR}+, 3.16)$ would increase the probability 


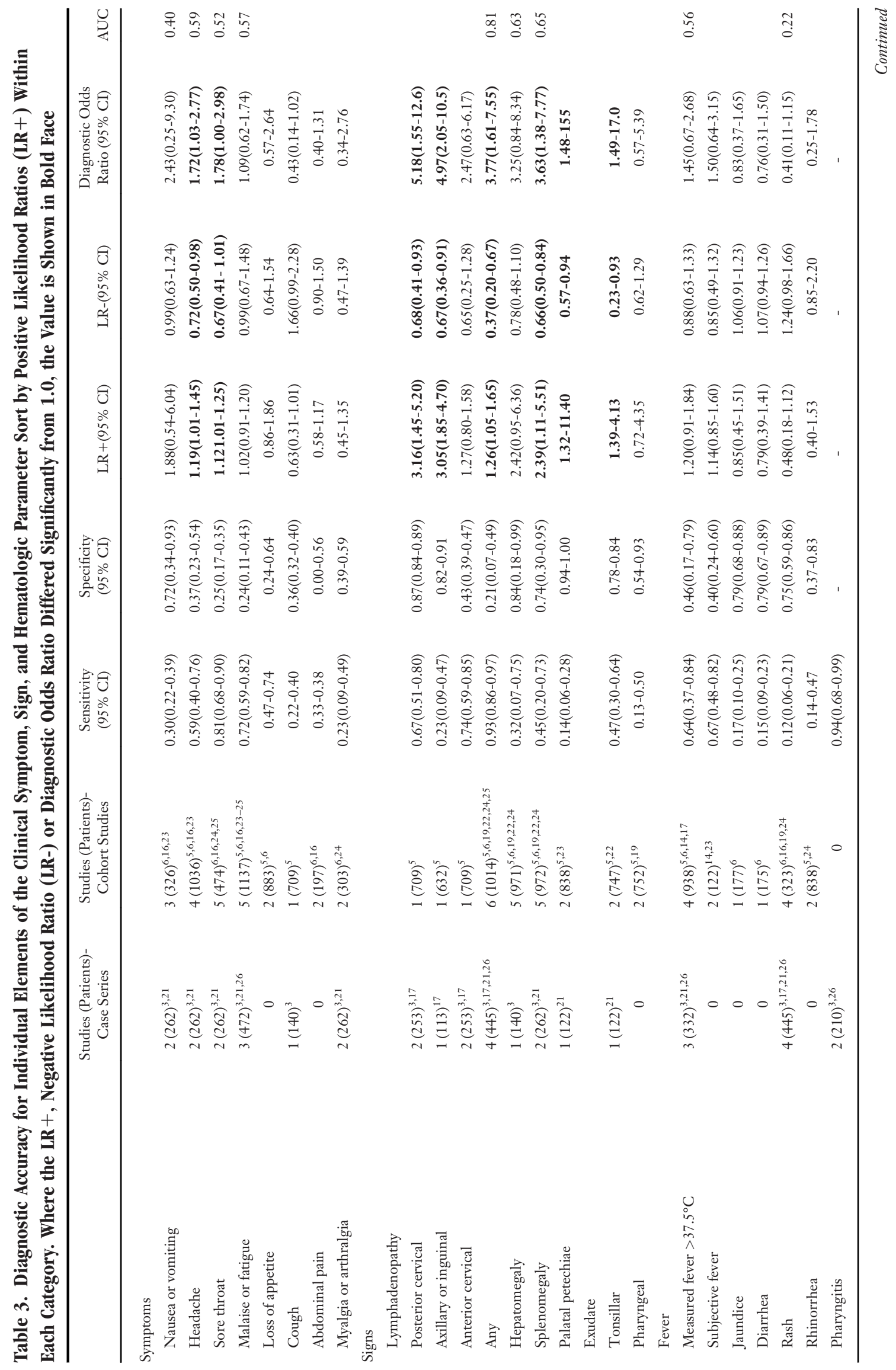




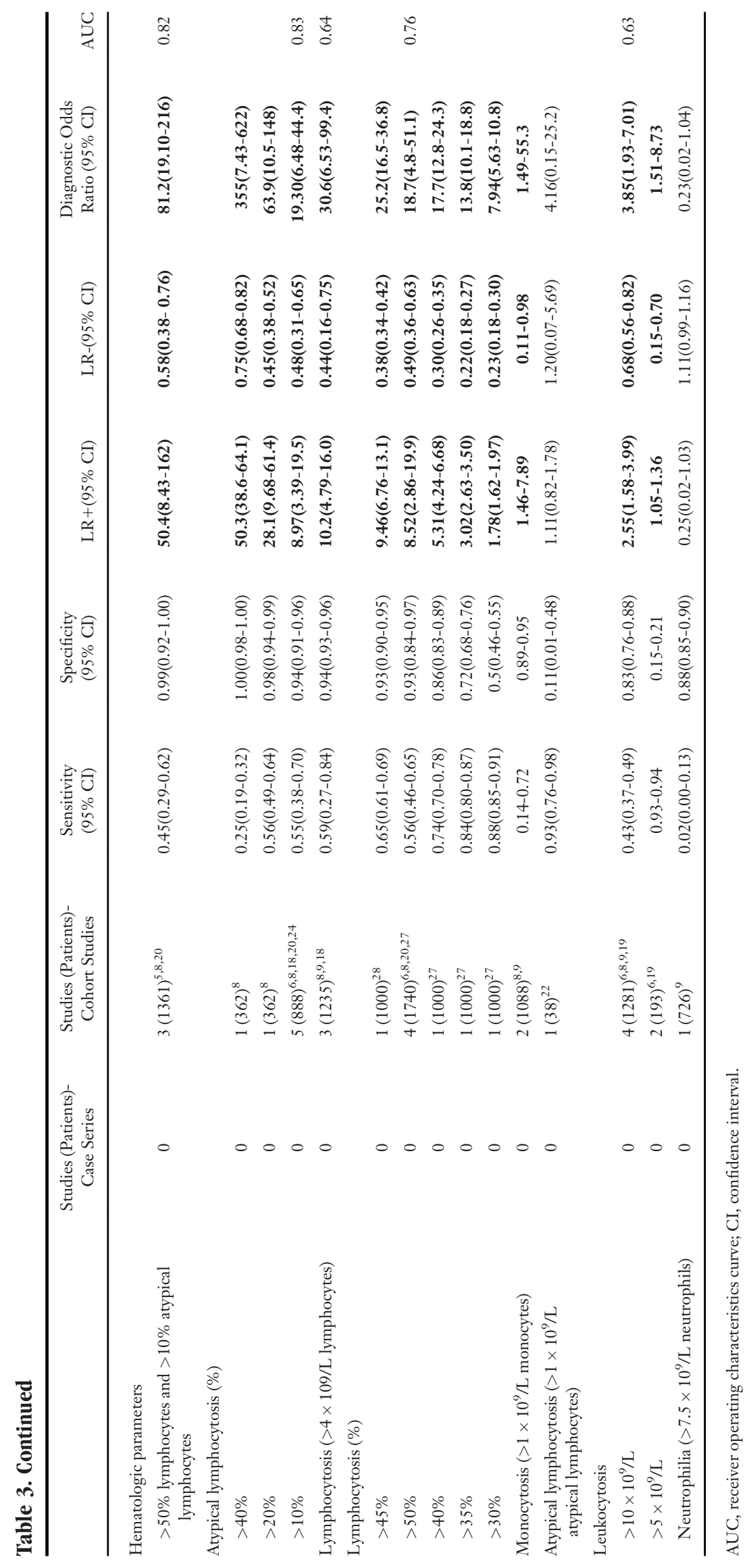


Figure 2. Receiver operative characteristic curve by cutoff values for (a) lymphocyte percentage and (b) atypical lymphocyte percentage.
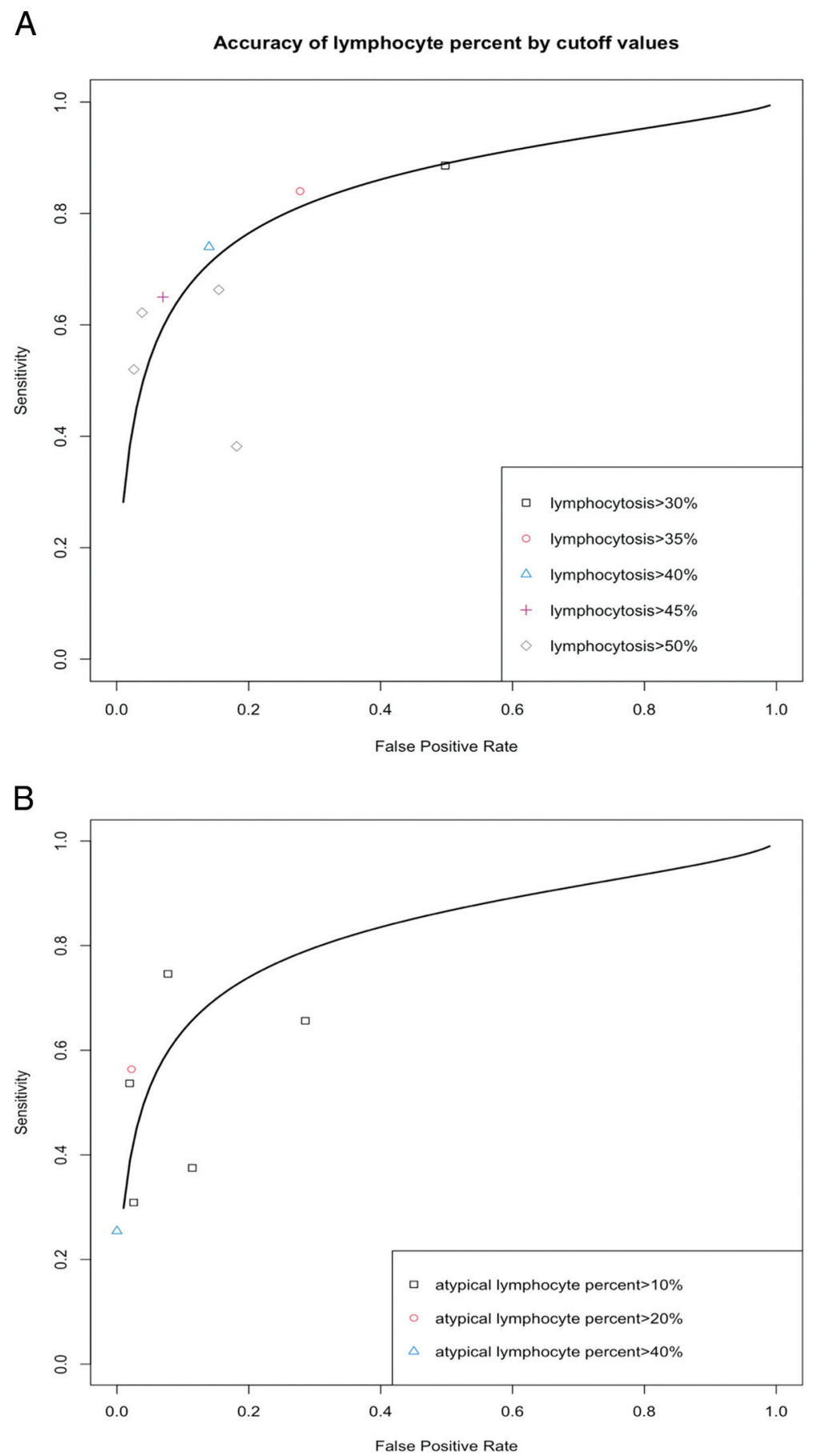

to $21 \%$. For hematologic parameters, a lymphocyte greater than $40 \%$ is an important diagnostic finding $(\mathrm{LR}+, 5.31)$ and would increase the probability of IM to $32 \%$. An atypical lymphocyte greater than $40 \%(\mathrm{LR}+, 50.3)$, and the combination of lymphocytes greater than $50 \%$ and atypical lymphocytes greater than $10 \%(\mathrm{LR}+, 50.4)$ would increase the probability of IM to $81 \%$. We found only 3 studies ${ }^{5,8,20}$ that assessed the accuracy of combinations of the lymphocyte and atypical lymphocyte percentages; no other studies were found that investigated the diagnostic accuracy of a 
Figure 3. Receiver operative characteristic curve stratified by the reference standard tests for (a) lymphocytes greater than $50 \%$ and (b) atypical lymphocytes greater than $10 \%$.

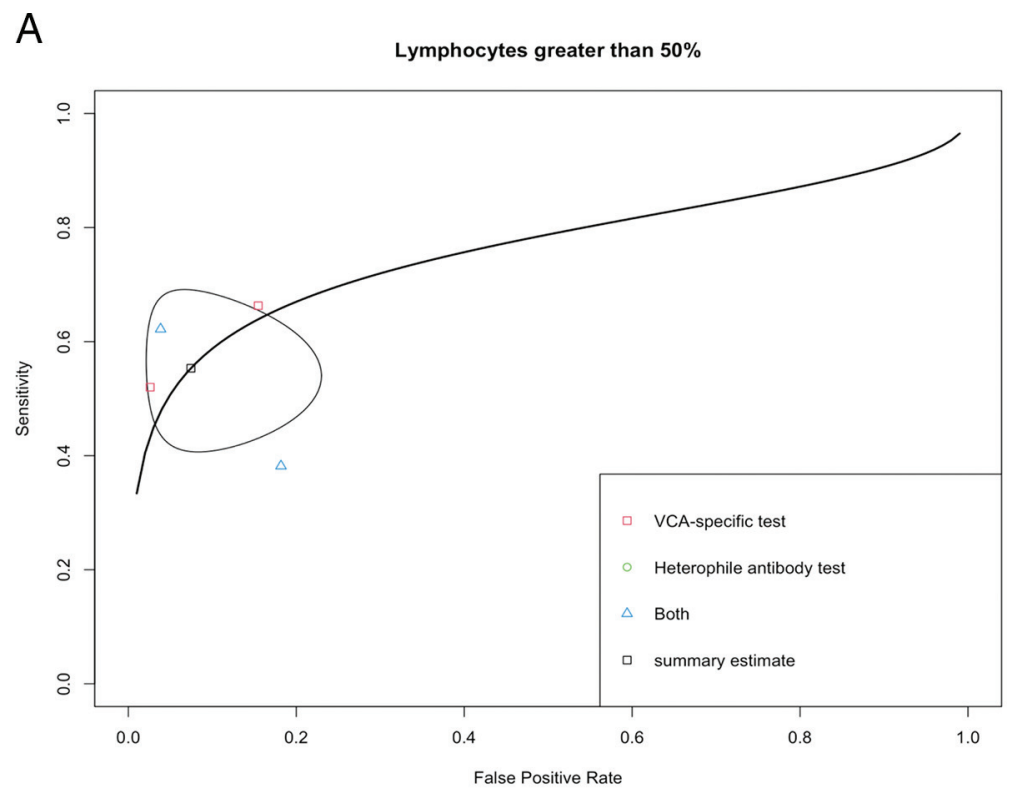

B

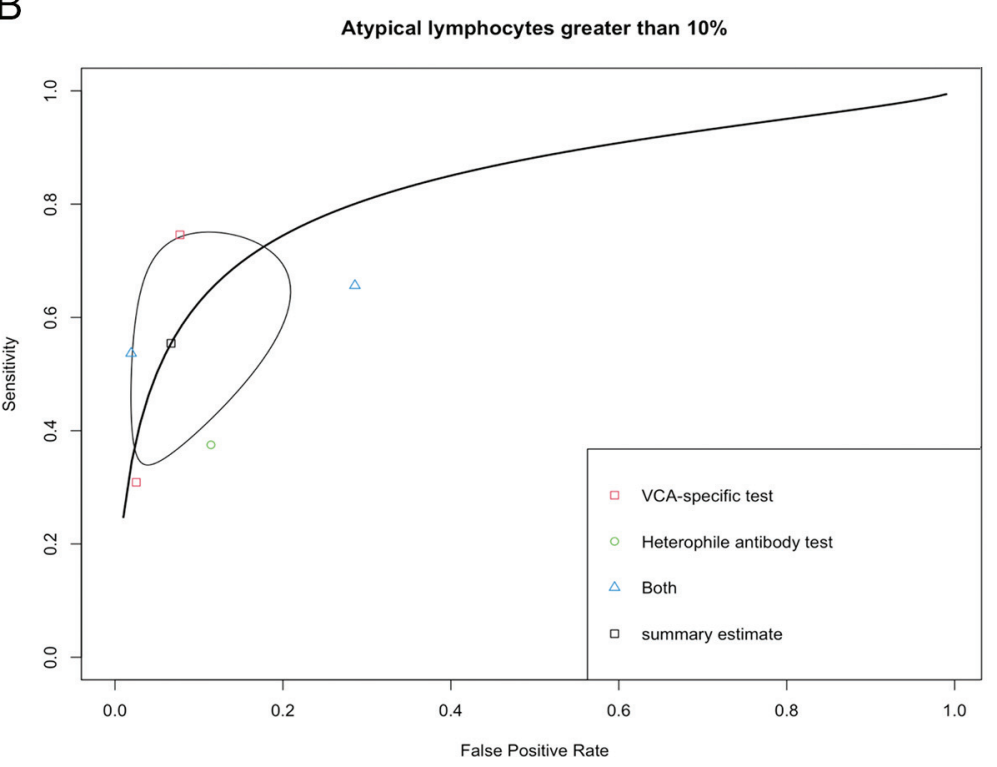

combination or combinations of signs, symptoms, and hematologic parameters.

\section{Strength and Limitation}

The strength of our study is that we included a comprehensive literature search and used a contemporary bivariate meta-analysis in the study. We found additional studies that addressed diagnostic accuracy compared with a previous review and provided a more precise summary estimation.
However, there are several limitations in this review. First, most studies did not specify the duration or severity of symptoms. Since the clinical findings varied by the progression of disease, it would be useful if the accuracy of the clinical findings were stratified by the duration from the onset of symptoms and signs. Second, some of the typical symptoms of IM, such as sore throat or pharyngitis, were considered as the inclusion criteria for the study population, and this might have biased the estimation of the individual value of these 
symptoms and signs for the diagnosis of IM due to incorporation bias.

\section{Conclusion}

In conclusion, while individual symptoms, signs, and hematologic parameters are important for the clinical diagnosis, only a few key signs and symptoms are significantly associated with IM. The likelihood of IM is appreciably increased by the presence of splenomegaly, palatal petechiae, posterior adenopathy, and axillary or inguinal lymphadenopathy, while it is significantly decreased by the absence of any lymphadenopathy. The most helpful hematologic parameters for diagnosing IM include lymphocytes greater than $4 \times 10^{9} / \mathrm{L}$ and greater than $40 \%$ to $50 \%$, atypical lymphocytes greater than $40 \%$, and a combination of lymphocytes greater than $50 \%$, and atypical lymphocytes greater than $10 \%$. Well-designed prospective studies are needed to investigate the role of the clinical findings and office-based tests among patients within a week of disease onset and evaluate the natural history of IM to help clinicians better understand the course of the disease.

To see this article online, please go to: http://jabfm.org/content/ 34/6/1141.full.

\section{References}

1. Henke CE, Kurland LT, Elveback LR. Infectious mononucleosis in Rochester, Minnesota, 1950 through 1969. Am J Epidemiol 1973;98:483-90.

2. Ebell MH, Call M, Shinholser J, Gardner J. Does this patient have infectious mononucleosis?: the rational clinical examination systematic review diagnosing infectious mononucleosis diagnosing infectious mononucleosis. Jama 2016;315:1502-9.

3. Rea TD, Russo JE, Katon W, Ashley RL, Buchwald DS. Prospective study of the natural history of infectious mononucleosis caused by Epstein-Barr virus. J Am Board Fam Pract 2001;14:234-42.

4. Hoagland RJ. The clinical manifestations of infectious mononucleosis. a report of two hundred cases. American Journal of Medical Sciences 1960;240:21-9.

5. Aronson MD, Komaroff AL, Pass TM, Ervin CT, Branch WT. Heterophil antibody in adults with sore throat: frequency and clinical presentation. Ann Intern Med 1982;96:505-8.

6. Grotto I, Mimouni D, Huerta M, et al. Clinical and laboratory presentation of EBV positive infectious mononucleosis in young adults. Epidemiol Infect 2003;131:683-9.

7. Rice L, Jung M. Neutrophilic leukocytosis, neutropenia, monocytosis, and monocytopenia. In: Hematology. Elsevier; 2018:675-81.
8. Brigden ML, Au S, Thompson S, Brigden S, Doyle $\mathrm{P}$, Tsaparas Y. Infectious mononucleosis in an outpatient population: diagnostic utility of 2 automated hematology analyzers and the sensitivity and specificity of Hoagland's criteria in heterophile-positive patients. Arch Pathol Lab Med 1999;123:875-81.

9. Biggs TC, Hayes SM, Bird JH, Harries PG, Salib RJ. Use of the lymphocyte count as a diagnostic screen in adults with suspected Epstein-Barr virus infectious mononucleosis. Laryngoscope 2013;123:2401-4.

10. Hutton B, Salanti G, Caldwell DM, et al. The PRISMA extension statement for reporting of systematic reviews incorporating network meta-analyses of health care interventions: checklist and explanations. Ann Intern Med 2015;162:777-84.

11. Whiting PF, Rutjes AW, Westwood ME, QUADAS-2 Group, et al. QUADAS-2: a revised tool for the quality assessment of diagnostic accuracy studies. Ann Intern Med 2011;155:529-36.

12. Reitsma J, Rutjes A, Whiting P, Vlassov V, Leeflang M. Assessing methodological quality. In: Deeks JJ, Bossuyt PM, Gatsonis C (editors), Cochrane Handbook for Systematic Reviews of Diagnostic Test Accuracy Version 1.0. 0. The Cochrane Collaboration, 2009. Available from: http://srdta cochrane org/. 2009.

13. Doebler P, Holling H. Meta-Analysis of Diagnostic Accuracy the mada: R package version 0.5.8. 2017.

14. Team RC. R: A language and environment for statistical computing. $R$ Foundation for Statistical Computing. 2013. [Database]

15. Metz CE. Basic principles of ROC analysis. Semin Nucl Med 1978;8:283-98.

16. Balfour HH, Jr., Holman CJ, Hokanson KM, et al. A prospective clinical study of Epstein-Barr virus and host interactions during acute infectious mononucleosis. J Infect Dis 2005;192:1505-12.

17. Sumaya CV, Ench Y. Epstein-Barr virus infectious mononucleosis in children. I. Clinical and general laboratory findings. Pediatrics 1985;75:1003-10.

18. Ventura KC, Hudnall SD. Hematologic differences in heterophile-positive and heterophile-negative infectious mononucleosis. Am J Hematol 2004;76:315-8.

19. Ginsburg CM, Henle W, Henle G, Horwitz CA. Infectious mononucleosis in children. Evaluation of Epstein-Barr virus-specific serological data. Jama 1977;237:781-5.

20. Fleisher G, Collins M, Fager S. Limitations of available tests for diagnosis of infectious mononucleosis. J Clin Microbiol 1983;17:619-24.

21. Chretien J, Esswein J, Holland W, McCauley C. Predictors of the duration of infectious mononucleosis. South Med J 1977;70:437-9.

22. Hossain A. Infectious mononucleosis and mononucleosis-like illnesses in children and adults in Saudi Arabia. J Trop Pediatr 1989;35:121-5. 
23. Llor C, Hernández M, Hernández S, Martínez T, Gómez FF. Validity of a point-of-care based on heterophile antibody detection for the diagnosis of infectious mononucleosis in primary care. Eur J Gen Pract 2012;18:15-21.

24. Krabbe S, Hesse J, Uldall P. Primary Epstein-Barr virus infection in early childhood. Arch Dis Child 1981;56:49-52.

25. Ho-Yen D, Martin KW. The relationship between atypical lymphocytosis and serological tests in infectious mononucleosis. J Infect 1981;3:324-31.

26. Gartzonika C, Vrioni G, Priavali E, Pappas G, Levidiotou S. Utility of real-time PCR in the diagnosis of primary Epstein-Barr virus infection. J Med Microb Diagn 2012;02:2161-0703.1000118.

27. Lennon P, O' Neill JP, Fenton JE, O’ Dwyer T. Challenging the use of the lymphocyte to white cell count ratio in the diagnosis of infectious mononucleosis by analysis of a large cohort of Monospot test results. Clin Otolaryngol 2010; 35:397-401.

28. Schooley R. Epstein-Barr virus infections, including infectious mononucleosis. Harrison's Principles of Internal Medicine 1987;699-703.

29. Horwitz CA, Henle W, Henle G, et al. Clinical and laboratory evaluation of elderly patients with heterophil-antibody positive infectious mononucleosis: Report of seven patients, ages 40 to 78 . Am J Med 1976;61:333-9.

30. Carter JW, Edson RS, Kennedy CC. Infectious mononucleosis in the older patient. Mayo Clinic Proc 1978;53(3):146-50.

31. Del CM, Pincus D. Incidence patterns of respiratory illness in Queensland estimated from sentinel general practice. Aust Fam Physician 1995;24: 625-9. 